\title{
Mild solutions to nonlocal impulsive differential inclusions governed by a noncompact semigroup
}

\section{Shaochun Ji}

Faculty of Mathematics and Physics, Huaiyin Institute of Technology, Huaian 223003, P. R. China.

Communicated by R. Saadati

\begin{abstract}
In this paper, we study the existence of mild solutions to impulsive differential inclusions with nonlocal conditions in general Banach spaces when operator semigroup is not compact. By using measure of noncompactness and multivalued analysis, we give some sufficient conditions on the existence results where the impulsive items and the nonlocal items are compact and Lipschitz continuous, respectively. An example concerning with the partial differential equation is also presented. (C)2017 all rights reserved.
\end{abstract}

Keywords: Differential inclusions, impulsive conditions, fixed point theorems, measure of noncompactness, nonlocal conditions.

2010 MSC: 34G10, 34A60.

\section{Introduction}

In this paper we are concerned with the following differential inclusions with nonlocal conditions and impulsive conditions

$$
\left\{\begin{array}{l}
u^{\prime}(t) \in A u(t)+F(t, u(t)), \quad t \in[0, b], t \neq t_{i}, \\
\Delta u\left(t_{i}\right)=u\left(t_{i}^{+}\right)-u\left(t_{i}^{-}\right)=I_{i}\left(u\left(t_{i}\right)\right), \quad i=1,2, \cdots, s, \\
u(0)=g(u),
\end{array}\right.
$$

where $A: D(A) \subseteq X \rightarrow X$ is the infinitesimal generator of a strongly continuous semigroup $T(t), t \geqslant 0$ in a Banach space $(X,\|\cdot\|), F$ is an upper-Carathéodory multifunction, $0=t_{0}<t_{1}<t_{2}<\cdots<t_{s}<t_{s+1}=b$, and $u\left(t_{i}^{+}\right)$and $u\left(t_{i}^{-}\right)$denote the right and the left limit of $u$ at $t_{i}$, respectively. $g, I_{i}$ are appropriate continuous functions to be specified later.

The theory of impulsive differential equations and impulsive differential inclusions has received much attention because of its wide applications in physics, biology, engineering, medical fields, and technology. The reason for this applicability arises from the fact that impulsive differential problems serve as an appropriate model for describing a process which cannot be described by the classical differential problems.

Email address: jiscmath@163. com (Shaochun Ji)

doi:10.22436/jnsa.010.02.14 
For more details on this theory and on its applications we refer to the monographs of Lakshmikantham et al. [18], Benchohra et al.[6], the papers of [1, 10, 14, 23] and the references therein. In [10], Cardinali et al. considered the impulsive semilinear differential inclusions under the assumptions of the measure of noncompactness with multivalued perturbations F. Moreover, Byszewski and Lakshmikantham [9] introduced the nonlocal Cauchy problems as the corresponding models that can describe the phenomena more accurately than the classical initial condition $\mathfrak{u}(0)=\mathfrak{u}_{0}$ alone. Therefore, it has been studied extensively under various conditions on $A$ and $F$ by several authors (see $[3,8,20])$. We remark that the main difficulty on nonlocal Cauchy problem is how to get the compactness of the solution operator at zero. Some methods are developed to deal with this difficulty in nonlocal problems, we refer the readers to the papers $[13,19,23,24]$. Fan et al. [13] discussed the nonlocal impulsive differential problem of single-valued case

$$
\left\{\begin{array}{l}
u^{\prime}(t)=A u(t)+f(t, u(t)), \quad t \in[0, b], t \neq t_{i}, \\
u(0)+g(u)=u_{0}, \\
\Delta u\left(t_{i}\right)=I_{i}\left(u\left(t_{i}\right)\right), \quad i=1,2, \cdots, s, 0<t_{1}<t_{2}<\cdots<t_{s}<b,
\end{array}\right.
$$

where semigroup $\mathrm{T}(\mathrm{t})$ is compact, and $\mathrm{g}$ is Lipschitz continuous, compact, and strongly continuous, respectively. Then Ji et al. [15] extended the results to impulsive differential inclusions and the conditions on $F$ and $g$ are weakened where $T(t)$ is compact and $g$ is only supposed to be continuous.

In this paper, under a noncompact assumption on the semigroup $T(t)$, we provide some theorems on the existence of mild solutions for impulsive differential inclusions (1.1) for the case that the functions $I_{i}$ and $g$ are compact and for the case that $I_{i}$ and $g$ are Lipschitz continuous. In fact, here the semigroup $T(t)$ is supposed to be equicontinuous and the Banach space $X$ is not separable. We get the existence results of impulsive differential inclusions (1.1) via measures of noncompactness and the fixed point theorems for multifunctions, which improve the results in $[7,15,19]$.

This paper is organized as follows. In Section 2, we recall some concepts and facts about the multifunctions and measure of noncompactness. In Sections 3 and 4, we obtain the existence of mild solutions to nonlocal impulsive problem (1.1) when the functions $I_{i}$ and $g$ are compact and Lipschitz continuous, respectively. At last, an example is presented to illustrate the application of our results.

\section{Preliminaries}

Throughout this paper, let $\mathbb{N}, \mathbb{R}$ and $\mathbb{R}^{+}$be the set of positive integers, real numbers and positive real numbers, respectively. We denote by $\mathrm{C}([0, b] ; X)$ the space of $X$-valued continuous functions on $[0, b]$ with the norm $\|x\|=\sup \{\|x(t)\|, t \in[0, b]\}$ and by $L^{1}([0, b] ; X)$ the space of $X$-valued Bochner integrable functions on $[0, b]$ with the norm $\|f\|_{L^{1}}=\int_{0}^{b}\|f(t)\| d t$. Define $\operatorname{PC}([0, b] ; X)=\{u:[0, b] \rightarrow X$ : $u$ is continuous at $t \neq t_{i}$ and left continuous at $t=t_{i}^{-}$, and the right limit $u\left(t_{i}^{+}\right)$exists, $\left.i=1, \cdots, s\right\}$. It is easy to verify that $P C([0, b] ; X)$ is a Banach space with the norm $\|u\|_{P C}=\sup \{\|u(t)\|, t \in[0, b]\}$.

Let $X$ and $Y$ be two Hausdorff topological spaces. We use the notations $P(Y)=\left\{A \in 2^{Y}: A \neq \varnothing\right\}$, $P_{c l}(Y)=\{A \in P(Y): A$ is closed $\}, P_{b}(Y)=\{A \in P(Y): A$ is bounded $\}, P_{c}(Y)=\{A \in P(Y): A$ is convex $\}$, $P_{c p}(Y)=\{A \in P(Y): A$ is compact $\}$, and $P_{c p, c}(Y)=\left\{A \in P(Y): A \in P_{c p}(Y) \cap P_{c}(Y)\right\}$.

A multivalued map $F: X \rightarrow P(Y)$ is said to be:

(i) convex (closed) valued if $F(x)$ is convex (closed) in $Y$ for all $x \in X$;

(ii) compact if $F(B)$ is relatively compact for every $B \in P_{b}(X)$.

$F: X \rightarrow P(Y)$ is said to be upper semi-continuous (u.s.c.) on $X$ if for each $x_{0} \in X$ the set $F\left(x_{0}\right)$ is a nonempty, closed subset of $Y$, and if for each open subset $K$ of $Y$ containing $F\left(x_{0}\right)$, there exists an open neighborhood $\Gamma$ of $x_{0}$ such that $\mathrm{F}(\Gamma) \subseteq \mathrm{K}$.

Lemma 2.1 ([17]). Assume that $\mathrm{D} \subset \mathrm{X}$ and $\mathrm{F}(\mathrm{x})$ is closed for all $\mathrm{x} \in \mathrm{D}$, then the following conclusions hold:

(i) if $\mathrm{F}$ is upper semi-continuous and $\mathrm{D}$ is closed, then $\mathrm{F}$ has a closed graph (i.e., $x_{n} \rightarrow x, y_{n} \rightarrow y, y_{n} \in F\left(x_{n}\right)$ imply $\mathrm{y} \in \mathrm{F}(\mathrm{x})$ ); 
(i) if $\overline{\mathrm{F}(\mathrm{D})}$ is compact and $\mathrm{D}$ is closed, then $\mathrm{F}$ is upper semi-continuous if and only if $\mathrm{F}$ has a closed graph.

We set

$$
\mathrm{S}_{\mathrm{F}}(\mathrm{u}):=\left\{\mathrm{f} \in \mathrm{L}^{1}([0, \mathrm{~b}] ; \mathrm{X}): \mathrm{f}(\mathrm{t}) \in \mathrm{F}(\mathrm{t}, \mathrm{u}(\mathrm{t})) \text { a.e. on }[0, \mathrm{~b}]\right\}
$$

and say $F$ has a fixed point if there is $x \in X$ such that $x \in F(x)$.

Definition 2.2. A function $u \in P C([0, b] ; X)$ is called a mild solution of impulsive differential inclusions (1.1) if

$$
u(t)=T(t) g(u)+\int_{0}^{t} T(t-s) f(s) d s+\sum_{0<t_{i}<t} T\left(t-t_{i}\right) I_{i}\left(u\left(t_{i}\right)\right),
$$

for all $t \in[0, b]$, where $f \in S_{F}(u)$.

Now we give some facts on measure of noncompactness, see Banas and Goebel [4].

Definition 2.3. Let $E^{+}$be the positive cone of an ordered Banach space $(E, \leqslant)$. A function $\Phi$ defined on the set of all bounded subsets of the Banach space $X$ with values in $E^{+}$is called a measure of noncompactness (in short MNC) on X if $\Phi(\overline{\operatorname{conv}} \Omega)=\Phi(\Omega)$ for all bounded subsets $\Omega \subset$ X, where $\overline{\operatorname{conv}} \Omega$ stands for the closed convex hull of $\Omega$.

The MNC $\Phi$ is said to be

(1) monotone if for all bounded subsets $\Omega_{1}, \Omega_{2}$ of $\mathrm{X}$ we have: $\left(\Omega_{1} \subseteq \Omega_{2}\right) \Rightarrow\left(\Phi\left(\Omega_{1}\right) \leqslant \Phi\left(\Omega_{2}\right)\right)$;

(2) nonsingular if $\Phi(\{a\} \cup \Omega)=\Phi(\Omega)$ for every $a \in X, \Omega \subset X$;

(3) regular if $\Phi(\Omega)=0$ if and only if $\Omega$ is relatively compact in X.

One of the most important examples of MNC is the Hausdorff measure of noncompactness $\beta(\cdot)$ defined by

$$
\beta(B)=\inf \{\varepsilon>0 ; B \text { has a finite } \varepsilon \text {-net in } X\},
$$

for each bounded subset $B$ in a Banach space $X$.

It is well-known that the Hausdorff measure of noncompactness $\beta$ enjoys the above properties and other properties.

Lemma 2.4 ([4]). Let X be a real Banach space and B, C $\subset$ X be bounded. Then the following properties are satisfied:

(1) $B$ is relatively compact if and only if $\beta(B)=0$;

(2) $\beta(B)=\beta(\bar{B})=\beta(\operatorname{conv}(B))$, where $\bar{B}$ and $\operatorname{conv}(B)$ mean the closure and convex hull of $B$, respectively;

(3) $\beta(\mathrm{B}) \leqslant \beta(\mathrm{C})$ when $\mathrm{B} \subseteq \mathrm{C}$;

(4) $\beta(B+C) \leqslant \beta(B)+\beta(C)$, where $B+C=\{x+y: x \in B, y \in C\}$;

(5) $\beta(B \cup C) \leqslant \max \{\beta(B), \beta(C)\}$;

(6) $\beta(\lambda \mathrm{B}) \leqslant|\lambda| \beta(\mathrm{B})$ for any $\lambda \in \mathbb{R}$;

(7) if the map $\mathrm{Q}: \mathrm{D}(\mathrm{Q}) \subseteq \mathrm{X} \rightarrow \mathrm{Z}$ is Lipschitz continuous with constant $\mathrm{k}$, then $\beta_{\mathrm{Z}}(\mathrm{QB}) \leqslant \mathrm{k} \beta(\mathrm{B})$ for any bounded subset $\mathrm{B} \subseteq \mathrm{D}(\mathrm{Q})$, where $\mathrm{Z}$ is a Banach space;

(8) if $\left\{W_{n}\right\}_{n=1}^{\infty}$ is a decreasing sequence of bounded closed nonempty subsets of $X$ and $\lim _{n \rightarrow \infty} \beta\left(W_{n}\right)=0$, then $\bigcap_{\mathrm{n}=1}^{\infty} \mathrm{W}_{\mathrm{n}}$ is nonempty and compact in $\mathrm{X}$.

Lemma 2.5 ([4]).

(1) If $\mathrm{W} \subset \mathrm{C}([0, \mathrm{~b}] ; \mathrm{X})$ is bounded, then $\beta(\mathrm{W}(\mathrm{t})) \leqslant \beta(\mathrm{W})$ for all $\mathrm{t} \in[0, \mathrm{~b}]$, where $\mathrm{W}(\mathrm{t})=\{\mathrm{u}(\mathrm{t}) ; \mathrm{u} \in$ $W\} \subset X$. Furthermore if $W$ is equicontinuous on $[0, b]$, then $\beta(W(t))$ is continuous on $[0, b]$ and $\beta(W)=$ $\sup \{\beta(W(t)), t \in[0, b]\}$. 
(2) If $\mathrm{W} \subset \mathrm{C}([0, \mathrm{~b}] ; \mathrm{X})$ is bounded and equicontinuous, then

$$
\beta\left(\int_{0}^{t} W(s) d s\right) \leqslant \int_{0}^{t} \beta(W(s)) d s,
$$

for all $\mathrm{t} \in[0, \mathrm{~b}]$, where $\int_{0}^{\mathrm{t}} \mathrm{W}(\mathrm{s}) \mathrm{d} s=\left\{\int_{0}^{\mathrm{t}} \mathrm{x}(\mathrm{s}) \mathrm{d} s: \mathrm{x} \in \mathrm{W}\right\}$.

Then the results of Lemma 2.5 are extended to the space PC $([0, \mathrm{~b}] ; \mathrm{X})$ by Ji and $\mathrm{Li}[16]$.

Lemma 2.6 ([16]). If $\mathrm{W} \subset \mathrm{PC}([0, \mathrm{~b}] ; \mathrm{X})$ is bounded, then $\beta(\mathrm{W}(\mathrm{t})) \leqslant \beta(\mathrm{W})$ for all $\mathrm{t} \in[0, \mathrm{~b}]$, where $\mathrm{W}(\mathrm{t})=$ $\{\mathrm{u}(\mathrm{t}) ; \mathrm{u} \in \mathrm{W}\} \subset \mathrm{X}$. Furthermore, suppose the following conditions are satisfied:

(1) $W$ is equicontinuous on $\mathrm{J}_{0}=\left[0, \mathrm{t}_{1}\right]$ and each $\mathrm{J}_{i}=\left(\mathrm{t}_{i}, \mathrm{t}_{i+1}\right], i=1, \cdots, \mathrm{s}$;

(2) $W$ is equicontinuous at $t=t_{i}^{+}, i=1, \cdots, s$.

Then $\sup _{t \in[0, b]} \beta(W(t))=\beta(W)$.

For any bounded subset $B \subset X$, we define by

$$
\beta_{0}(B)=\sup \left\{\beta\left(\left\{x_{\mathfrak{n}}: n \geqslant 1\right\}\right):\left\{x_{\mathfrak{n}}\right\}_{\mathfrak{n}=1}^{\infty} \text { is a sequence in } B\right\} .
$$

From [4] we know that

$$
\beta_{0}(B) \leqslant \beta(B) \leqslant 2 \beta_{0}(B) .
$$

If $X$ is a separable space, we have $\beta_{0}(B)=\beta(B)$.

Lemma 2.7 ([17, Theorem 4.2.2]). Let $\left\{\mathrm{f}_{\mathrm{n}}\right\}_{\mathrm{n}=1}^{+\infty}$ be a sequence of functions in $\mathrm{L}^{1}([0, \mathrm{~b}] ; \mathrm{X})$. Assume that there exist $\mu, \eta \in \mathrm{L}^{1}\left([0, \mathrm{~b}] ; \mathbb{R}^{+}\right)$satisfying

$$
\sup _{n \geqslant 1}\left\|f_{n}(t)\right\| \leqslant \mu(t) \text { and } \beta\left(\left\{f_{n}(t)\right\}_{n=1}^{+\infty}\right) \leqslant \eta(t) \text { a.e. } t \in[0, b] .
$$

Then for all $\mathrm{t} \in[0, \mathrm{~b}]$, we have

$$
\beta\left(\left\{\int_{0}^{t} T(t-s) f_{n}(s) d s: n \geqslant 1\right\}\right) \leqslant 2 M \int_{0}^{t} \eta(s) d s,
$$

where $M=\sup _{t \in[0, b]}\|\mathrm{T}(\mathrm{t})\|$.

The semigroup $T(t)$ is said to be equicontinuous if $\{T(t) x: x \in B\}$ is equicontinuous at $t>0$ for any bounded subset $B \subset X$ (see $[5,24])$. Obviously if $T(t)$ is a compact semigroup or an analytic semigroup, it must be equicontinuous. And the converse of the relation usually is not correct. We give the following assumptions on $T(t)$, $F$.

(HA) The semigroup $\{T(t): t \geqslant 0\}$ generated by $A$ is equicontinuous. Moreover, there exists a positive number $M$ such that $M=\sup _{0 \leqslant t \leqslant b}\|T(t)\|$ (see [21]).

(HF1) $F$ is an upper Carathéodory multifunction, i.e., for every $x \in X$ the multifunction $F(\cdot, x):[0, b] \rightarrow$ $P_{c p, c}(X)$ admits a strongly measurable selector; for a.e. $t \in[0, b]$ the multifunction $F(t, \cdot): X \rightarrow$ $\mathrm{P}_{c p, c}(X)$ is upper semi-continuous. And for each $u \in P C([0, b] ; X)$, the set $S_{F}(u)$ is nonempty.

Lemma 2.8 ([22]). Let $\mathrm{X}$ be a Banach space and $\mathrm{F}$ a multifunction satisfying assumption (HF1). Let

$$
\Gamma: \mathrm{L}^{1}([0, \mathrm{~b}] ; \mathrm{X}) \rightarrow \mathrm{C}([0, \mathrm{~b}] ; \mathrm{X})
$$

be a linear continuous mapping. Then the operator

$$
\Gamma \circ S_{\mathrm{F}}: \mathrm{C}([0, \mathrm{~b}] ; X) \rightarrow \mathrm{P}_{\mathrm{cl}, \mathrm{c}}(\mathrm{C}([0, \mathrm{~b}] ; X)), x \rightarrow\left(\Gamma \circ \mathrm{S}_{\mathrm{F}}\right)(\mathrm{x}):=\Gamma\left(\mathrm{S}_{\mathrm{F}}(\mathrm{x})\right)
$$

is a closed graph operator in $\mathrm{C}([0, \mathrm{~b}] ; \mathrm{X}) \times \mathrm{C}([0, \mathrm{~b}] ; \mathrm{X})$. 
Lemma 2.9 ([16]). If the hypothesis (HA) is satisfied, i.e., $\{\mathbf{T}(\mathrm{t}): \mathrm{t} \geqslant 0\}$ is equicontinuous, and $\eta \in \mathrm{L}^{1}\left([0, \mathrm{~b}] ; \mathbb{R}^{+}\right)$, then the set $\left\{\int_{0}^{\mathrm{t}} \mathrm{T}(\mathrm{t}-\mathrm{s}) \mathrm{u}(\mathrm{s}) \mathrm{ds}:\|\mathrm{u}(\mathrm{s})\| \leqslant \eta(\mathrm{s})\right.$ for a.e. $\left.\mathrm{s} \in[0, \mathrm{~b}]\right\}$ is equicontinuous for $\mathrm{t} \in[0, \mathrm{~b}]$.

The following fixed point theorems are important in our proof.

Lemma 2.10 ([2]). Let $\mathrm{D}$ be a nonempty, closed, and convex subset of a completely Hausdorff locally convex linear topological space and let $\mathrm{F}: \mathrm{D} \rightarrow \mathrm{P}(\mathrm{D})$ be an upper semi-continuous and compact map with $\mathrm{F}(\mathrm{x})$ a nonempty, closed, and convex subset of $\mathrm{D}$. Then $\mathrm{F}$ has a fixed point in $\mathrm{D}$.

A multivalued function $F: D \subset X \rightarrow P(X)$ is said to be strict $\beta$-contraction if it maps bounded sets into bounded sets and there exists a constant $0 \leqslant k<1$ such that $\beta(F(B)) \leqslant k \beta(B)$ for all bounded sets $B \subset D$.

Lemma 2.11 ([12]). Let $\mathrm{X}$ be a Banach space, $\mathrm{D} \subset \mathrm{X}$ bounded, closed and convex, $\mathrm{F}: \mathrm{D} \rightarrow \mathrm{P}(\mathrm{D})$ upper semicontinuous and a strict $\beta$-contraction, and $\mathrm{F}(\mathrm{x})$ closed convex for all $\mathrm{x} \in \mathrm{D}$. Then $\mathrm{F}$ has at least a fixed point in D.

\section{Results under compact conditions}

In this section, we give some results for impulsive nonlocal problem (1.1) when the functions $g$ and $I_{i}$ are compact, which map bounded sets into relatively compact sets.

We define the solution map $\mathrm{G}: \mathrm{PC}([0, \mathrm{~b}] ; \mathrm{X}) \rightarrow \mathrm{P}(\mathrm{PC}([0, \mathrm{~b}] ; \mathrm{X}))$ by

$$
\begin{aligned}
& (\mathrm{Gu})(\mathrm{t}) \\
& \quad=\left\{v \in \mathrm{PC}([0, \mathrm{~b}] ; \mathrm{X}): v(\mathrm{t})=\mathrm{T}(\mathrm{t}) \mathrm{g}(\mathrm{u})+\int_{0}^{\mathrm{t}} \mathrm{T}(\mathrm{t}-\mathrm{s}) \mathrm{f}(\mathrm{s}) \mathrm{d} s+\sum_{0<\mathrm{t}_{\mathrm{i}}<\mathrm{t}} \mathrm{T}\left(\mathrm{t}-\mathrm{t}_{\mathrm{i}}\right) \mathrm{I}_{\mathrm{i}}\left(\mathrm{u}\left(\mathrm{t}_{\mathrm{i}}\right)\right), \mathrm{f} \in \mathrm{S}_{\mathrm{F}}(\mathrm{u})\right\}
\end{aligned}
$$

with

$$
\begin{aligned}
& \left(G_{1} u\right)(t)=T(t) g(u), \\
& \left(G_{2} u\right)(t)=\left\{v \in C([0, b] ; X): v(t)=\int_{0}^{t} T(t-s) f(s) d s, f \in S_{F}(u)\right\}, \\
& \left(G_{3} u\right)(t)=\sum_{0<t_{i}<t} T\left(t-t_{i}\right) I_{i}\left(u\left(t_{i}\right)\right)
\end{aligned}
$$

for all $t \in[0, b]$. It is easy to see that $u$ is the mild solution of the problem (1.1) if and only if $u$ is a fixed point of the map G.

We list the following hypotheses.

(HF2) There exists a function $\mathrm{m}(\mathrm{t}) \in \mathrm{L}\left([0, \mathrm{~b}] ; \mathbb{R}^{+}\right)$and a nondecreasing function $\mathrm{q}: \mathbb{R}^{+} \rightarrow \mathbb{R}^{+}$such that for a.e. $t \in[0, b], x \in X$,

$$
\|F(t, x)\|:=\sup \{\|y\|: y \in F(t, x)\} \leqslant m(t) q(\|x\|) .
$$

(HF3) There exists a constant $L>0$ such that for any bounded set $\mathrm{D} \subset \mathrm{X}$,

$$
\beta(F(t, D)) \leqslant L \beta(D),
$$

for a.e. $t \in[0, b]$.

(Hg1) $\mathrm{g}: \mathrm{PC}([0, \mathrm{~b}] ; \mathrm{X}) \rightarrow \mathrm{X}$ is continuous, compact and there exists a nondecreasing function $\gamma: \mathbb{R}^{+} \rightarrow \mathbb{R}^{+}$ such that

$$
\|g(u)\| \leqslant \gamma\left(\|u\|_{P C}\right) .
$$


(HI1) For $i=1, \cdots, s, I_{i}: X \rightarrow X$ is continuous, compact and there exists a nondecreasing function $l_{i}: \mathbb{R}^{+} \rightarrow \mathbb{R}^{+}$such that for $x \in X$,

$$
\left\|\mathrm{I}_{i}(\mathrm{x})\right\| \leqslant \mathrm{l}_{\mathrm{i}}(\|x\|) .
$$

Theorem 3.1. Assume that the hypotheses (HA), (HF1), (HF2), (HF3), (Hg1), (HI1) are satisfied, then the nonlocal impulsive problem (1.1) has at least one mild solution on $[0, \mathrm{~b}]$, provided that the condition

$$
\lim _{x \rightarrow+\infty} \sup \frac{M\left[\gamma(x)+q(x)\|m\|_{L^{1}}+\sum_{i=1}^{s} l_{i}(x)\right]}{x}<1
$$

is satisfied.

Proof. We turn the problem (1.1) into a fixed point problem by using solution map G defined in equation (3.1). Subsequently, we will prove $\mathrm{G}$ has a fixed point by using Lemma 2.10. The proof will be given in several steps.

Step 1. Set $W_{0}=\left\{x \in P C([0, b] ; X):\|x\| \leqslant r_{0}\right\}$. Then $W_{0} \subset P C([0, b] ; X)$ is bounded and convex. We claim that $G W_{0} \subset W_{0}$. Let $x \in W_{0}, y \in G(x)$. From hypotheses (HF2), (Hg1) and (HI1), we have

$$
\begin{aligned}
\|y(t)\| & \leqslant M \gamma\left(\|x\|_{P C}\right)+M \int_{0}^{b} m(s) q\left(\|x\|_{P C}\right) d s+M \sum_{i=1}^{s} l_{i}\left(\|x\|_{P C}\right) \\
& \leqslant M \gamma\left(r_{0}\right)+M\|m\|_{L^{1}} q\left(r_{0}\right)+M \sum_{i=1}^{s} l_{i}\left(r_{0}\right),
\end{aligned}
$$

for $t \in[0, b]$. By condition (3.2), we know that there exists a constant $r_{0}>0$ such that

$$
M \gamma\left(r_{0}\right)+M\|m\|_{L^{1}} q\left(r_{0}\right)+M \sum_{i=1}^{s} l_{i}\left(r_{0}\right) \leqslant r_{0} .
$$

Therefore, $\mathrm{G}\left(\mathrm{W}_{0}\right) \subset \mathrm{W}_{0}$.

Step 2. We show that $G W_{0}$ is equicontinuous on $J_{0}=\left[0, t_{1}\right], J_{i}=\left(t_{i}, t_{i+1}\right]$ and is also equicontinuous at $t=t_{i}^{+}, i=1, \cdots, s$. Indeed, we only need to prove that $\left.G W_{0}\right|_{\left[t_{1}, t_{2}\right]}$ is equicontinuous on $\left[t_{1}, t_{2}\right]$, as the cases for other subintervals are the same. For $u \in W_{0}, t_{1} \leqslant s<t \leqslant t_{2}$, we have, using the semigroup property, that

$$
\|T(t) g(u)-T(s) g(u)\|=\|T(s)[T(t-s)-T(0)] g(u)\| \leqslant M\|[T(t-s)-T(0)] g(u)\| .
$$

Thus, $G_{1} W_{0}$ is equicontinuous on $\left[t_{1}, t_{2}\right]$ due to the compactness of $g$ and the strong continuity of $T(\cdot)$. The same idea can be used to prove the equicontinuity of $G_{3} W_{0}$ on $\left[t_{1}, t_{2}\right]$. That is, for $u \in W_{0}, t_{1} \leqslant s<t \leqslant t_{2}$, we have

$$
\left\|T\left(t-t_{1}\right) I_{1}\left(u\left(t_{1}\right)\right)-T\left(s-t_{1}\right) I_{1}\left(u\left(t_{1}\right)\right)\right\| \leqslant M\left\|[T(t-s)-T(0)] I_{1}\left(u\left(t_{1}\right)\right)\right\|,
$$

which implies the equicontinuity of $G_{3} W_{0}$ on $\left[t_{1}, t_{2}\right]$ due to the compactness of $I_{1}$ and the strong continuity of $\mathrm{T}(\cdot)$.

Now we consider the functions in $G_{2} W_{0}=\left\{\int_{0}^{t} T(t-s) f(s) d s, f \in S_{F}(u), u \in W_{0}\right\}$. As

$$
\|f(t)\| \leqslant\|F(t, u(t))\| \leqslant m(t) q\left(\|u\|_{P C}\right) \leqslant m(t) q\left(r_{0}\right),
$$

and $\mathrm{m}(\mathrm{t}) \in \mathrm{L}^{1}\left([0, \mathrm{~b}] ; \mathbb{R}^{+}\right)$, from Lemma 2.9, we know $\mathrm{G}_{2} \mathrm{~W}_{0}$ is equicontinuous on $[0, \mathrm{~b}]$. Therefore, we have that the functions in $\left.G W_{0}\right|_{\left[t_{1}, t_{2}\right]}=\left.\left(G_{1}+G_{2}+G_{3}\right) W_{0}\right|_{\left[t_{1}, t_{2}\right]}$ are equicontinuous on $\left[t_{1}, t_{2}\right]$.

Step 3. For any $x_{0} \in W_{0}$, define by

$$
\mathrm{W}_{1}=\overline{\operatorname{conv}}\left\{\mathrm{GW}_{0}, \mathrm{x}_{0}\right\}, \mathrm{W}_{\mathrm{n}}=\overline{\operatorname{conv}}\left\{\mathrm{GW} \mathrm{W}_{\mathrm{n}-1}, \mathrm{x}_{0}\right\},
$$

where $\overline{\text { conv }}$ means the closure of convex hull, $n=2,3, \cdots$. We know that $W_{n+1} \subset W_{n}$ for $n=1,2, \cdots$ 
as $W_{1} \subset W_{0}$, and $W_{1}, W_{2}, \cdots, W_{n}, \cdots$ are nonempty, closed, bounded, convex, and equicontinuous. Thus $\left\{W_{n}\right\}$ is a decreasing sequence of subsets of $P C([0, b] ; X)$. We set

$$
W=\cap_{n=1}^{\infty} W_{n},
$$

then $W$ is a nonempty, closed, bounded, convex and equicontinuous subset of $P C([0, b] ; X)$.

From (2.1), we have, for any $\varepsilon>0$, there are sequences $\left\{u_{k}\right\}_{k=1}^{\infty} \subset W_{0}$ and $\left\{f_{k}\right\}_{k=1}^{\infty} \subset L^{1}([0, b] ; X)$ such that $f_{k} \in S_{F}\left(u_{k}\right)$ for all $k \geqslant 1$ and

$$
\beta\left(\left\{\int_{0}^{t} T(t-s) f(s) d s, f \in S_{F}(u), u \in W_{0}\right\}\right) \leqslant 2 \beta\left(\left\{\int_{0}^{t} T(t-s) f_{k}(s) d s: k \geqslant 1\right\}\right)+\varepsilon .
$$

Then, due to Lemma 2.7, we have

$$
\begin{aligned}
\beta\left(G_{2} W_{0}(t)\right) & =\beta\left(\left\{\int_{0}^{t} T(t-s) f(s) d s, f \in S_{F}(u), u \in W_{0}\right\}\right) \\
& \leqslant 2 \beta\left(\left\{\int_{0}^{t} T(t-s) f_{k}(s) d s: k \geqslant 1\right\}\right)+\varepsilon \\
& \leqslant 4 M \int_{0}^{t} \beta\left(\left\{f_{k}(s): k \geqslant 1\right\}\right) d s+\varepsilon \\
& \leqslant 4 M \int_{0}^{t} \beta\left(\left\{F(s, u(s)), u \in W_{0}\right\}\right) d s+\varepsilon \\
& \leqslant 4 M \int_{0}^{t} L \beta\left(W_{0}(s)\right) d s+\varepsilon \\
& \leqslant 4 M L t \beta\left(W_{0}\right)+\varepsilon .
\end{aligned}
$$

Since $\varepsilon>0$ is arbitrary, it follows from the above inequality that

$$
\beta\left(\left\{\int_{0}^{t} T(t-s) f(s) d s, f \in S_{F}(u), u \in W_{0}\right\}\right) \leqslant 4 M \operatorname{Lt} \beta\left(W_{0}\right) .
$$

Noticing the compactness of $g, I_{i}$ and (3.3), we have

$$
\begin{aligned}
\beta\left(W_{1}(t)\right) \leqslant & \beta\left(T(t) g\left(W_{0}\right)\right)+\beta\left(\left\{\int_{0}^{t} T(t-s) f(s) d s, f \in S_{F}(u), u \in W_{0}\right\}\right) \\
& \left.+\beta\left(\left\{\sum_{0<t_{i}<t} T\left(t-t_{i}\right) I_{i}\left(u\left(t_{i}\right)\right), u \in W_{0}\right)\right\}\right) \\
\leqslant & \beta\left(\left\{\int_{0}^{t} T(t-s) f(s) d s, f \in S_{F}(u), u \in W_{0}\right\}\right) \\
\leqslant & 4 M L t \beta\left(W_{0}\right),
\end{aligned}
$$

for $t \in[0, b]$. Further,

$$
\begin{aligned}
\beta\left(W_{2}(t)\right) \leqslant & \beta\left(T(t) g\left(W_{1}\right)\right)+\beta\left(\left\{\int_{0}^{t} T(t-s) f(s) d s, f \in S_{F}(u), u \in W_{1}\right\}\right) \\
& \left.+\beta\left(\left\{\sum_{0<t_{i}<t} T\left(t-t_{i}\right) I_{i}\left(u\left(t_{i}\right)\right), u \in W_{1}\right)\right\}\right) \\
\leqslant & \beta\left(\left\{\int_{0}^{t} T(t-s) f(s) d s, f \in S_{F}(u), u \in W_{1}\right\}\right)
\end{aligned}
$$




$$
\begin{aligned}
& \leqslant 4 M \int_{0}^{t} L \beta\left(W_{1}(s)\right) d s \\
& \leqslant 4 M \int_{0}^{t} L \cdot 4 M \operatorname{Ls} \beta\left(W_{0}\right) d s \\
& \leqslant \frac{(4 M L)^{2} t^{2}}{2 !} \beta\left(W_{0}\right),
\end{aligned}
$$

for $t \in[0, b]$. We can continue this iterative procedure and get that

$$
\beta\left(W_{n}(t)\right) \leqslant \frac{(4 M L t)^{n}}{n !} \beta\left(W_{0}\right),
$$

for $t \in[0, b]$. As $W_{n}$ is equicontinuous on each subinterval $\left[t_{i}, t_{i+1}\right], i=1, \cdots, s$, by Lemma 2.6, we have that

$$
\beta\left(W_{n}\right)=\sup _{t \in[0, b]} \beta\left(W_{n}(t)\right) \leqslant \frac{(4 M L b)^{n}}{n !} \beta\left(W_{0}\right) .
$$

By the fact that $\frac{(4 M L b)^{n}}{n !} \rightarrow 0$ as $n \rightarrow \infty$, we have $\beta\left(W_{n}\right) \rightarrow 0$. According to Lemma 2.4 (8), we get that $W=\cap_{n=1}^{\infty} W_{n}$ is compact in $\mathrm{PC}([0, \mathrm{~b}] ; X)$.

Step 4. Let us verify $G(W) \subset W$. In fact, $G(W) \subset G\left(W_{n}\right) \subset \overline{\operatorname{conv}}\left\{G W_{n}, x_{0}\right\}=W_{n+1}$ for every $n \geqslant 1$. Then $\mathrm{G}(\mathrm{W}) \subset \bigcap_{n=2}^{\infty} W_{n}$. On the other hand, $W_{n} \subset W_{1}$ for every $n \geqslant 1$. Then we have

$$
\mathrm{G}(\mathrm{W}) \subset \bigcap_{\mathrm{n}=2}^{\infty} \mathrm{W}_{\mathrm{n}}=\bigcap_{\mathrm{n}=1}^{\infty} \mathrm{W}_{\mathrm{n}}=\mathrm{W} .
$$

At this point, we get that $G: W \rightarrow W$ is a compact map.

Step 5. Now, we show that $\left.\mathrm{G}\right|_{W}: \mathrm{W} \rightarrow \mathrm{P}(\mathrm{W})$ has a closed graph and is upper semi-continuous. Let $\left(u_{m}\right)_{m \in \mathbb{N}},\left(v_{m}\right)_{m \in \mathbb{N}} \subset W, u_{m} \rightarrow u, v_{m} \in G\left(u_{m}\right), v_{m} \rightarrow v$ in $\operatorname{PC}([0, b] ; X)$. Then there exists a sequence $\left\{f_{m}\right\}_{m=1}^{\infty} \subset L^{1}([0, b] ; X), f_{m} \in S_{F}\left(u_{m}\right)$ for $m \geqslant 1$, such that

$$
v_{m}(t)=T(t) g\left(u_{m}\right)+\int_{0}^{t} T(t-s) f_{m}(s) d s+\sum_{0<t_{i}<t} T\left(t-t_{i}\right) I_{i}\left(u_{m}\left(t_{i}\right)\right),
$$

for all $t \in[0, b]$. Consider the linear operator $\Gamma: \mathrm{L}^{1}([0, \mathrm{~b}] ; \mathrm{X}) \rightarrow \mathrm{C}([0, \mathrm{~b}] ; \mathrm{X})$ defined as $(\Gamma \mathrm{f})(\mathrm{t})=\int_{0}^{\mathrm{t}} \mathrm{T}(\mathrm{t}-$ $s) f(s) d s$. Obviously, $\Gamma$ is linear and continuous. From equation (3.4), we have

$$
v_{m}(\cdot)-T(\cdot) g\left(u_{m}\right)-\sum_{0<t_{i}<\cdot} T\left(\cdot-t_{i}\right) I_{i}\left(u_{m}\left(t_{i}\right)\right) \in \Gamma \circ S_{F}\left(u_{m}\right) .
$$

Then from Lemma 2.8, we get that $\Gamma \circ S_{\mathrm{F}}(\cdot)$ is a closed graph operator. Since $u_{\mathrm{m}} \rightarrow u$ and $v_{\mathrm{m}} \rightarrow v$, we obtain that

$$
v(\cdot)-\mathrm{T}(\cdot) \mathrm{g}(\mathrm{u})-\sum_{0<\mathrm{t}_{i}<\cdot} \mathrm{T}\left(\cdot-\mathrm{t}_{\mathrm{i}}\right) \mathrm{I}_{\mathrm{i}}\left(\mathrm{u}\left(\mathrm{t}_{\mathrm{i}}\right)\right) \in \Gamma \circ \mathrm{S}_{\mathrm{F}}(\mathrm{u}),
$$

from the continuity of $T(t), g, I_{i}$. That is,

$$
v(t)-T(t) g(u)-\sum_{0<t_{i}<t} T\left(t-t_{i}\right) I_{i}\left(u\left(t_{i}\right)\right)=\int_{0}^{t} T(t-s) f(s) d s,
$$

for some $f \in S_{F}(u)$. Therefore, $G$ has a closed graph, which implies that $G$ has closed values on $\mathrm{PC}([0, b] ; X)$. Moreover, as $\overline{\mathrm{G}(W)}$ is compact and $W$ is closed, from Lemma 2.1, we get that $G$ is upper semi-continuous.

From Steps 1-5, the multifunction $\left.\mathrm{G}\right|_{W}: W \rightarrow P(W)$ is an upper semi-continuous, compact map with $\mathrm{G}(\mathrm{x})$ a nonempty, closed, convex subset of $W$. Due to Lemma 2.10, there is $u \in W$ such that $u \in G(u)$ and $u$ is the mild solution to the nonlocal impulsive problem (1.1). This completes the proof of Theorem 3.1. 
Remark 3.2. If multifunction $F(\cdot)$ is compact or Lipschitz continuous, then $L$ in hypothesis (HF3) becomes zero or a Lipschitz constant. In Benchohra et al. [7], the existence and topological structures of mild solutions to impulsive differential inclusions are discussed when the Banach space $X$ is separable. Here by using the estimation to Hausdorff measure of noncompactness, we get the existence results for nonlocal impulsive differential inclusions (1.1) when $X$ is not separable and the semigroup $T(t)$ is only equicontinuous without the compactness assumption.

\section{Results under Lipschitz conditions}

Now we study the existence result of problem (1.1) when the functions $g$ and $I_{i}$ are Lipschitz continuous.

In this section, we list the following hypotheses:

(Hg2) $\mathrm{g}: \mathrm{PC}([0, \mathrm{~b}] ; X) \rightarrow X$ is Lipschitz continuous with Lipschitz constant $k$, that is, $\|\mathrm{g}(\mathrm{u})-\mathrm{g}(v)\| \leqslant$ $k\|u-v\|$ for $u, v \in \operatorname{PC}([0, b] ; X)$. And there exists a nondecreasing function $\gamma: \mathbb{R}^{+} \rightarrow \mathbb{R}^{+}$such that

$$
\|g(u)\| \leqslant \gamma\left(\|u\|_{P C}\right)
$$

(HI2) $I_{i}: X \rightarrow X$ is Lipschitz continuous with Lipschitz constant $k_{i}$, that is,

$$
\left\|I_{i}(x)-I_{i}(y)\right\| \leqslant k_{i}\|x-y\|
$$

for $x, y \in X, i=1, \cdots, s$. And there exists a nondecreasing function $l_{i}: \mathbb{R}^{+} \rightarrow \mathbb{R}^{+}$such that for $x \in X$,

$$
\left\|\mathrm{I}_{\mathrm{i}}(\mathrm{x})\right\| \leqslant \mathrm{l}_{\mathrm{i}}(\|x\|) .
$$

Theorem 4.1. Assume that the hypotheses (HA), (HF1), (HF2), (HF3), (Hg2), (HI2) are satisfied, then the nonlocal impulsive problem (1.1) has at least one mild solution on $[0, \mathrm{~b}]$, provided that the condition (3.2) and

$$
M k+4 M L b+M \sum_{i=1}^{s} k_{i}<1
$$

are satisfied.

Proof. We denote by $W_{0}=\{u \in P C([0, b] ; X):\|u(t)\| \leqslant r$ for all $t \in[0, b]\}$. Then $W_{0}$ is a bounded, closed and convex subset of $P C([0, b] ; X)$. The functions $G, G_{1}, G_{2}, G_{3}$ are defined as equation (3.1).

According to Theorem 3.1, we have proved that $\mathrm{GW}_{0} \subset \mathrm{W}_{0}$ and $\mathrm{G}$ has a closed graph on $\mathrm{PC}([0, \mathrm{~b}] ; \mathrm{X})$ with closed convex values. Now we shall prove that $G: W_{0} \rightarrow P\left(W_{0}\right)$ is upper semi-continuous and is a strict $\beta$-contraction.

First, we prove that $G$ is a strict $\beta$-contraction on $W_{0}$. For $u, v \in P C([0, b] ; X)$, we have $G_{1}(u), G_{1}(v) \in$ $\mathrm{C}([0, \mathrm{~b}] ; \mathrm{X})$, and from hypothesis $(\mathrm{Hg} 2)$,

$$
\begin{aligned}
\left\|G_{1}(u)-G_{1}(v)\right\| C & =\sup _{t \in[0, b]}\left\|\left(G_{1} u\right)(t)-\left(G_{1} v\right)(t)\right\| \\
& =\sup _{t \in[0, b]}\|T(t) g(u)-T(t) g(v)\| \\
& \leqslant M\|g(u)-g(v)\| \\
& \leqslant M k\|u-v\| P C .
\end{aligned}
$$

Then from Lemma 2.4 (7), for every bounded set $B \subset W_{0}$, we get

$$
\beta\left(G_{1} B\right) \leqslant M k \beta(B) .
$$


From formula (2.1), for any $\varepsilon>0$ and $B \subset W_{0}$, there are sequences $\left\{\boldsymbol{u}_{k}\right\}_{k=1}^{\infty} \subset B$ and $\left\{\boldsymbol{f}_{k}\right\}_{k=1}^{\infty} \subset$ $\mathrm{L}^{1}([0, b] ; X)$ such that $f_{k} \in S_{F}\left(u_{k}\right)$ for all $k \geqslant 1$ and

$$
\beta\left(\left\{\int_{0}^{t} T(t-s) f(s) d s, f \in S_{F}(u), u \in B\right\}\right) \leqslant 2 \beta\left(\left\{\int_{0}^{t} T(t-s) f_{k}(s) d s: k \geqslant 1\right\}\right)+\varepsilon .
$$

As $T(t)$ is equicontinuous on $[0, b]$, by Lemma 2.9, we know that $G_{2} W_{0} \subset C([0, b] ; X)$ is equicontinuous on $[0, b]$. Then due to Lemma 2.5 and Lemma 2.7, for every set $B \subset W_{0}$, we have

$$
\begin{aligned}
\beta\left(G_{2} B\right) & =\sup _{t \in[0, b]} \beta\left(G_{2} B(t)\right) \\
& \leqslant \sup _{t \in[0, b]} 2 \beta\left(\left\{\int_{0}^{t} T(t-s) f_{k}(s) d s: k \geqslant 1\right\}\right)+\varepsilon \\
& \leqslant \sup _{t \in[0, b]} 4 M \int_{0}^{t} \beta\left(\left\{f_{k}(s): k \geqslant 1\right\}\right) d s+\varepsilon \\
& \leqslant \sup _{t \in[0, b]} 4 M \int_{0}^{t} \beta(\{F(s, u(s)), u \in B\}) d s+\varepsilon \\
& \leqslant \sup _{t \in[0, b]} 4 M \int_{0}^{t} L \beta(B(s)) d s+\varepsilon \\
& \leqslant 4 M L b \beta(B)+\varepsilon .
\end{aligned}
$$

Since $\varepsilon>0$ is arbitrary, it follows from the above inequality that

$$
\beta\left(G_{2} B\right) \leqslant 4 M \operatorname{Lb} \beta(B) .
$$

As $I_{i}: X \rightarrow X$ is Lipschitz continuous, we have, for $u, v \in W_{0}$,

$$
\left\|G_{3} u-G_{3} v\right\|_{P C}=\sup _{t \in[0, b]}\left\|\left(G_{3} u\right)(t)-\left(G_{3} v\right)(t)\right\| \leqslant M \sum_{i=1}^{s} k_{i}\|u-v\|_{P C} .
$$

So for $\mathrm{B} \subset \mathrm{W}_{0}$, we have

$$
\beta\left(G_{3} B\right) \leqslant M \sum_{i=1}^{s} k_{i} \beta(B) .
$$

From (4.2), (4.3), and (4.4), we obtain, for $B \subset W_{0}$,

$$
\begin{aligned}
\beta(G B) & \leqslant \beta\left(G_{1} B\right)+\beta\left(G_{2} B\right)+\beta\left(G_{3} B\right) \\
& \leqslant M k \beta(B)+4 M L b \beta(B)+M \sum_{i=1}^{s} k_{i} \beta(B) \\
& \leqslant\left(M k+4 M L b+M \sum_{i=1}^{s} k_{i}\right) \beta(B) .
\end{aligned}
$$

Noting (4.1), $M k+4 M L b+M \sum_{i=1}^{s} k_{i}<1$, then $G$ is a strict $\beta$-contraction on $W_{0}$.

Next, we shall prove that $G$ is upper semi-continuous. From Theorem 2 in [11], we know that $G_{2}(u)=$ $\left\{v \in C([0, b] ; X): v(t)=\int_{0}^{t} T(t-s) f(s) d s, f \in S_{F}(u)\right\}$ is upper semi-continuous. Let $\left\{u_{n}\right\}_{n=1}^{\infty}$ be a sequence in $\operatorname{PC}([0, b] ; X)$ with $\lim _{n \rightarrow \infty} u_{n}=u$ in $\operatorname{PC}([0, b] ; X)$. And we have

$$
\left\|\left(G_{1} u_{n}+G_{3} u_{n}\right)-\left(G_{1} u+G_{3} u\right)\right\|_{P C} \leqslant M\left[\left\|g\left(u_{n}\right)-g(u)\right\|+\sum_{i=1}^{s}\left\|I_{i}\left(u_{n}\left(t_{i}\right)\right)-I_{i}\left(u\left(t_{i}\right)\right)\right\|\right] .
$$


Then by the continuity of $g$ and $I_{i}$, we can deduce $G_{1}(u)+G_{3}(u)$ is continuous and hence

$$
\mathrm{G}(\mathrm{u})=\mathrm{G}_{1}(\mathrm{u})+\mathrm{G}_{2}(\mathrm{u})+\mathrm{G}_{3}(\mathrm{u})
$$

is upper semi-continuous on $\mathrm{PC}([0, \mathrm{~b}] ; \mathrm{X})$. Notice that $W_{0}$ is a closed subset of $\mathrm{PC}([0, \mathrm{~b}] ; \mathrm{X})$, so $\mathrm{G}$ is upper semi-continuous on $W_{0}$.

Now we get that $G: W_{0} \rightarrow P\left(W_{0}\right)$ is upper semi-continuous and a strict $\beta$-contraction, and $G(u)$ is closed and convex for all $u \in W_{0}$. Due to Lemma 2.11, there is $u \in W_{0}$ such that $u \in G u$. Clearly $u$ is a mild solution for the nonlocal impulsive problem (1.1). This completes the proof.

\section{An example}

As an application of Theorems 3.1 and 4.1, we discuss the following impulsive differential inclusions with nonlocal conditions:

$$
\left\{\begin{array}{l}
\frac{\partial}{\partial t} \omega(t, x) \in \frac{\partial^{2}}{\partial x^{2}} \omega(t, x)+F(t, \omega(t, x)), \quad 0 \leqslant t \leqslant b, \quad 0 \leqslant x \leqslant \pi \\
\omega(t, 0)=\omega(t, \pi)=0, \\
\omega\left(t_{i}^{+}, x\right)-\omega\left(t_{i}^{-}, x\right)=I_{i}\left(\omega\left(t_{i}, x\right)\right), \quad i=1,2, \cdots, s, \\
\omega(0, x)=g(\omega(t, x)) .
\end{array}\right.
$$

Take $\mathrm{X}=\mathrm{L}^{2}[0, \pi]$ and the operator $\mathrm{A}: \mathrm{D}(\mathrm{A}) \subseteq \mathrm{X} \rightarrow \mathrm{X}$ defined by $\mathrm{A} z=z^{\prime \prime}$, with

$$
\mathrm{D}(\mathrm{A})=\left\{z \in X: z, z^{\prime} \text { are absolutely continuous, } z^{\prime \prime} \in X, z(0)=z(\pi)=0\right\} .
$$

From Pazy [21], we know that $A$ is the infinitesimal generator of an analytic semigroup $T(t), t \geqslant 0$. This implies that $A$ satisfies the condition (HA).

Let $0<\mathrm{t}_{1}<\mathrm{t}_{2}<\cdots<\mathrm{t}_{\mathrm{s}}<\mathrm{b}, 0<\mathrm{s}_{1}<\mathrm{s}_{2}<\cdots<\mathrm{s}_{\mathrm{q}}<\mathrm{b}, \mathrm{c}_{\mathrm{j}} \in \mathbb{R}(j=1, \cdots, \mathrm{q}), \mathrm{h}(\cdot) \in \mathrm{L}^{1}([0, \mathrm{~b}] ; \mathbb{R})$, $\alpha_{i}>0$ and $\rho_{i} \in C([0, \pi] \times[0, \pi], \mathbb{R})$ for $i=1, \cdots$, s. We assume that the following conditions hold:

(1) $\mathrm{F}:[0, \mathrm{~b}] \times \mathrm{X} \rightarrow \mathrm{P}(\mathrm{X})$ is Carathéodory upper semi-continuous defined by

$$
\mathrm{F}(\mathrm{t}, z)(x)=\mathrm{F}(\mathrm{t}, z(x)), \quad 0 \leqslant \mathrm{t} \leqslant \mathrm{b}, 0 \leqslant x \leqslant \pi,
$$

and hypotheses (HF2) and (HF3) hold.

(2) $g(\omega(t, \xi))=\sum_{j=1}^{q} c_{j} \omega\left(s_{j}, \xi\right), \omega \in P C([0, b] ; X)$.

(3) $g(\omega(t, \xi))=\int_{0}^{b} h(s) \lg (1+|\omega(s, \xi)|) d s, \omega \in P C([0, b] ; X)$.

(4) $\mathrm{I}_{\mathfrak{i}}(\mathrm{u}(\xi))=\frac{1}{\alpha_{i}|\mathfrak{u}(\xi)|+\mathrm{t}_{i}}, u \in X, 1 \leqslant i \leqslant s$.

(5) $I_{i}(u(\xi))=\int_{0}^{\pi} \rho_{i}(\xi, y) \cos ^{2}(u(y)) d y, u \in X, 1 \leqslant i \leqslant s$.

Under the above assumptions, the partial differential equation (5.1) can be reformulated as the abstract problem (1.1). Then we obtain that

Conclusion 1. Under the conditions (1), (3), and (5), the assumptions in Theorem 3.1 are satisfied for large $r_{0}>0$. Therefore, the corresponding system (1.1) has at least a mild solution.

Conclusion 2. Under the conditions (1), (2), and (4), the assumptions in Theorem 4.1 are satisfied for large $r_{0}>0$. Therefore, the corresponding system (1.1) has at least a mild solution. 


\section{Acknowledgment}

Research is supported by the Natural Science Foundation of Jiangsu Province (No. BK20150415), the National Natural Science Foundation of China (No. 11601178) and the NSF of Jiangsu Education Committee (No. 14KJB110001).

\section{References}

[1] N. Abada, M. Benchohra, H. Hammouche, Existence and controllability results for nondensely defined impulsive semilinear functional differential inclusions, J. Differential Equations, 246 (2009), 3834-3863. 1

[2] R. P. Agarwal, M. Meehan, D. O'Regan, Fixed point theory and applications, Cambridge Tracts in Mathematics, Cambridge University Press, Cambridge, (2001). 2.10

[3] S. Aizicovici, V. Staicu, Multivalued evolution equations with nonlocal initial conditions in Banach spaces, NoDEA Nonlinear Differential Equations Appl., 14 (2007), 361-376. 1

[4] J. Banaś, K. Goebel, Measures of noncompactness in Banach spaces, Lecture Notes in Pure and Applied Mathematics, Marcel Dekker, Inc., New York, (1980). 2, 2.4, 2.5, 2

[5] V. Barbu, Nonlinear semigroups and differential equations in Banach spaces, Translated from the Romanian, Editura Academiei Republicii Socialiste România, Bucharest; Noordhoff International Publishing, Leiden, (1976). 2

[6] M. Benchohra, J. Henderson, S. K. Ntouyas, Impulsive differential equations and inclusions, Contemporary Mathematics and Its Applications, Hindawi Publishing Corporation, New York, (2006). 1

[7] M. Benchohra, J. J. Nieto, A. Ouahab, Impulsive differential inclusions involving evolution operators in separable Banach spaces, Ukrainian Math. J., 64 (2012), 991-1018. 1, 3.2

[8] I. Benedetti, N. V. Loi, L. Malaguti, Nonlocal problems for differential inclusions in Hilbert spaces, Set-Valued Var. Anal., 22 (2014), 639-656. 1

[9] L. Byszewski, V. Lakshmikantham, Theorem about the existence and uniqueness of a solution of a nonlocal abstract Cauchy problem in a Banach space, Appl. Anal., 40 (1990), 11-19. 1

[10] T. Cardinali, P. Rubbioni, Impulsive semilinear differential inclusions: topological structure of the solution set and solutions on non-compact domains, Nonlinear Anal., 69 (2008), 73-84. 1

[11] J.-F. Couchouron, M. Kamenskii, An abstract topological point of view and a general averaging principle in the theory of differential inclusions, Nonlinear Anal., 42 (2000), 1101-1129. 4

[12] K. Deimling, Nonlinear functional analysis, Springer-Verlag, Berlin, (1985). 2.11

[13] Z.-B. Fan, G. Li, Existence results for semilinear differential equations with nonlocal and impulsive conditions, J. Funct. Anal., 258 (2010), 1709-1727. 1

[14] E. Hernández, D. O’Regan, On a new class of abstract impulsive differential equations, Proc. Amer. Math. Soc., 141 (2013), 1641-1649. 1

[15] S.-C. Ji, G. Li, Existence results for impulsive differential inclusions with nonlocal conditions, Comput. Math. Appl., 62 (2011), 1908-1915. 1

[16] S.-C. Ji, G, Li, A unified approach to nonlocal impulsive differential equations with the measure of noncompactness, Adv. Difference Equ., 2012 (2012), 14 pages. 2, 2.6, 2.9

[17] M. Kamenskii, V. Obukhovskii, P. Zecca, Condensing multivalued maps and semilinear differential inclusions in Banach spaces, De Gruyter Series in Nonlinear Analysis and Applications, Walter de Gruyter \& Co., Berlin, (2001). 2.1, 2.7

[18] V. Lakshmikantham, D. D. Baĭnov, P. S. Simeonov, Theory of impulsive differential equations, Series in Modern Applied Mathematics, World Scientific Publishing Co., Inc., Teaneck, NJ, (1989). 1

[19] J. Liang, J. H. Liu, T.-J. Xiao, Nonlocal impulsive problems for nonlinear differential equations in Banach spaces, Math. Comput. Modelling, 49 (2009), 798-804. 1

[20] S. K. Ntouyas, P. C. Tsamatos, Global existence for semilinear evolution equations with nonlocal conditions, J. Math. Anal. Appl., 210 (1997), 679-687. 1

[21] A. Pazy, Semigroups of linear operators and applications to partial differential equations, Applied Mathematical Sciences, Springer-Verlag, New York, (1983). 2, 5

[22] H. R. Thieme, "Integrated semigroups" and integrated solutions to abstract Cauchy problems, J. Math. Anal. Appl., 152 (1990), 416-447. 2.8

[23] J.-R. Wang, A. G. Ibrahim, M. Fečkan, Nonlocal impulsive fractional differential inclusions with fractional sectorial operators on Banach spaces, Appl. Math. Comput., 257 (2015), 103-118. 1

[24] X.-M. Xue, Nonlocal nonlinear differential equations with a measure of noncompactness in Banach spaces, Nonlinear Anal., 70 (2009), 2593-2601. 1, 2 